\title{
SUICÍDIO E INTERNET: ANÁLISE DE RESULTADOS EM
} FERRAMENTAS DE BUSCA

\author{
SUICIDIO E INTERNET: ANÁLISIS DE RESULTADOS EN HERRAMIENTAS \\ DE BÚSQUEDA \\ SUICIDE AND INTERNET: ANALYSIS OF RESULTS IN SEARCH TOOLS
}

Juliana Oliveira Gomes

Faculdade Estácio de Sá, Juiz de Fora, MG e Faculdade de Minas, Muriaé/MG, Brasil

Makilim Nunes Baptista

Universidade São Francisco, Itatiba/SP, Brasil

Adriana Munhoz Carneiro

Instituto de Psiquiatria, Faculdade de Medicina da Universidade de São Paulo/SP, Brasil

Hugo Ferrari Cardoso

Universidade Sagrado Coração, Bauru/SP e Fundação Educacional “Dr Raul Bauab”, Jaú/SP, Brasil

\section{RESUMO}

Embora seja ainda um assunto incipiente no Brasil, a mídia pode exercer influência sobre diferentes atitudes dos sujeitos e, especificamente, sobre suicídio. Este estudo buscou replicar, com adaptações, uma pesquisa inglesa sobre conteúdos relacionados ao suicídio acessados na internet. Foram utilizadas, em três sites de busca, doze expressões-chave, como por exemplo, suicídio; métodos de suicídio; suicídio indolor; suicídio rápido entre outras. Foram analisadas as dez primeiras indicações de cada pesquisa; retirando-se os resultados repetidos, a amostra foi composta por 209 sites. A análise do conteúdo das páginas eletrônicas se pautou em 19 critérios de classificação, sendo os de maior frequência os sites acadêmicos; os de conteúdo religioso; o que retratam o suicídio em termos elegantes; e os sites mantidos por psicólogos ou profissionais de saúde. Como conclusão, observou-se que, diferente do descrito no artigo original, no Brasil não foram encontradas páginas com conteúdo pró-suicídio, promovendo, encorajando ou facilitando atos suicidas.

Palavras-chave: suicídio; mídia; internet.

\section{RESUMEN}

Aunque sea un asunto incipiente en Brasil, los medios de comunicación pueden ejercer influencia sobre diferentes actitudes de los sujetos, y específicamente sobre el suicidio. Se buscó reproducir una investigación inglesa con adaptaciones sobre contenidos relacionados al suicidio que fueron obtenidos en internet. Fueron utilizados tres sitios web de búsqueda y doce expresiones claves, como suicidio; métodos de suicidio; suicidio indoloro; suicidio rápido entre otras. Fueron analizadas las diez primeras indicaciones de cada investigación, cuja muestra eran 209 sitios web, y se retiraron los resultados repetidos. El análisis de contenido de los sitios web siguió 19 criterios de clasificación, siendo los de mayor frecuencia los académicos, de contenido religioso, los que colocan el suicidio de forma elegante, y los sitios mantenidos por psicólogos o profesionales de salud. Se concluyó que, a diferencia del artículo original, en Brasil no fueron encontradas páginas con contenidos pro-suicidio, promoviendo, enalteciendo o facilitando actos suicidas.

Palabras clave: suicidio; medios de comunicación; internet.

\section{ABSTRACT}

The media can influence people in different attitudes and, particularly, towards suicide. Although it's a still incipient subject in Brazil, this study aimed to replicate, with some adaptations, a British research work on the accessed suicide-related websites contents. Twelve keywords were used in three search engines, for example, suicide; methods of suicide; painless suicide and quick suicide. Only the first 10 statements were considered in each search, which resulted in a sample of 209 websites, after removing the doubled ones. The analysis of these webpage contents was guided by 19 criteria of categorization: those with higher frequencies were the academic websites, followed by the religious, the ones that portray suicide in fashionable terms and by those websites maintained by psychologists and health professionals. The findings show that, unlike the data disclosed by the original article, in Brazil there were no pro-suicide webpages that promote, encourage or facilitate suicide acts.

Keywords: suicide; media; internet. 
O suicídio é caracterizado como um ato de autolesão; portanto, como uma ação intencional de matar a si mesmo (Organização Mundial da Saúde OMS, 1993). Este ato constitui um grande problema de saúde pública, além de gerar dificuldades para aqueles que perderam um membro familiar por tais circunstâncias, e para a equipe médica que eventualmente o estivesse atendendo (OMS, 2000; Sun, Long, Boore, \& Tsao, 2005). De acordo com dados da Organização Mundial de Saúde (World Health Organization - WHO, 2003), a cada ano aproximadamente um milhão de pessoas morrem no mundo em decorrência de suicídio, principalmente na faixa etária compreendida entre 15 e 44 anos. Isso fez com que o suicídio se tornasse a terceira causa de morte mais freqüente na população jovem e adulta.

Quando se observa a taxa global de mortalidade, os dados indicam a ocorrência de um suicídio a cada 40 segundos (WHO, 2000a). Com relação às estimativas estatísticas, $90 \%$ dos suicídios se relaciona com transtornos mentais diversos, sendo que um dos mais frequentes é o transtorno depressivo maior (Sadock \& Sadock, 2007). No entanto, Andriessen (2006) alerta e expõe que, em alguns países, existem discrepâncias quanto às taxas de suicídio e às suas possíveis causas. $\mathrm{O}$ autor cita Portugal, por exemplo, que tem uma das maiores taxas de suicídios indeterminados, de forma que estes excedem as taxas divulgadas. O autor também atenta para a possibilidade dos casos em que o suicídio possa estar encoberto por causas como doenças, uso de drogas, acidentes, dificultando, assim, uma estatística real. Logo, pode-se hipotetizar que existam mais casos de suicídio do que os registrados ou, ainda, que as ideações suicidas possam ser encobertas pela própria família e pelo hospital onde a morte resultante de ideação suicida passa a ser classificada de outra forma.

Na Irlanda, Corcoran, Arensman e O'Mahony (2006) realizaram uma pesquisa com o objetivo de comparar os registros de morte e as datas de ocorrência, levando em conta a demora em fornecer registros precisos. Isso porque casos configurados como morte externa - tal como o suicídio - podem levar meses (ou até anos) para obter seu registro, por necessitarem de inquéritos, o que também dificulta a validade das estatísticas locais. Dessa forma, foram analisados os dados do Vital Statistics Section, provenientes da Central of Statistics Office referentes aos anos de 1987 a 2003. Os resultados demonstraram uma discrepância entre $\mathrm{o}$ ano de ocorrência e o ano de registro, e que essa diferença aumentava ao longo do tempo, chegando a aproximadamente $20 \%$ nos últimos anos pesquisados. Esse artigo, tal como o de Andriessen (2006), atenta para a necessidade de um maior controle dos dados.
No Brasil, dados epidemiológicos demonstram que a taxa de suicídio encontra-se na faixa de 4,1 por 100.000 habitantes (WHO, 2000b). MelloSantos, Bertolote e Wang (2005) descreveram as taxas de suicídio brasileiras em um intervalo de 20 anos, mediante dados disponibilizados pelo Departamento de Informática do Sistema Único de Saúde (DATASUS). Em números absolutos, o Brasil encontrou-se entre os 10 países com maiores taxas de mortes, passando de seis mil ao ano. Foi percebido que a taxa de suicídio no Brasil cresceu $20 \%$ e que, nesse período, os homens se suicidaram de duas a quatro vezes mais que mulheres, sendo que e o grupo mais expressivo variou de 15 a 24 anos.

Kliemann (2007) verificou a epidemiologia de óbitos em Florianópolis, de 2001 a 2005, mediante a revisão de 7.184 laudos, analisando variáveis como idade, sexo, dia de óbito, dia da semana, procedência e local de ocorrência. Os resultados indicaram que do total, 671 dos casos de morte corresponderam a suicídio, havendo destaque para a faixa etária entre 20 e 49 anos, relativa a $66,2 \%$ dos óbitos. Quanto ao sexo, observou-se que $85,4 \%$ dos casos de suicídio correspondiam ao sexo masculino, com maiores índices às quintas-feiras $(16,7 \%)$ e aos domingos $(15,8 \%)$. Os dados também foram dispostos de acordo com as estações do ano, verificando-se uma maior ocorrência no verão e nos meses de agosto $(10,8 \%)$ e março $(9,6 \%)$.

Parente, Soares, Araújo, Cavalcante e Monteiro (2007) realizaram um estudo de caracterização do suicídio em Teresina, região nordeste do país. Foram avaliados 1.254 laudos dos anos 2000 a 2005, com relação a variáveis como sexo, idade, estado civil, ocupação, procedência/zona, mês de ocorrência e meios utilizados. Dos laudos avaliados, 244 casos referiam-se a mortes por suicídio, sendo predominante o sexo masculino $(71,3 \%)$, e a faixa etária compreendida entre 20 a 29 anos $(43,2 \%$ em 2001; $31,6 \%$ em 2003 e 40,5\% em 2005). Nos outros anos, os autores observaram maior prevalência da faixa entre 10 e 19 anos (30,9\%) em 2004; de 30 a 39 anos, em 2002 (26,3\%); e em idosos, no ano de 2000, (28,1\%). Quanto ao estado civil, observou-se prevalência de suicídio entre solteiros nos anos de 2001 (59,1\%); 2003 (55,3\%); 2004 (56,4\%); e 2005 (67,6\%). Em relação à ocupação, $23,8 \%$ eram estudantes, seguidos por aposentados $(10,7 \%)$ e desempregados $(9,0 \%)$. Por fim, os métodos mais utilizados por esta amostra foram enforcamento $(66 \%)$, armas de fogo $(13,1 \%)$ e envenenamento $(11,9 \%)$.

Mais recentemente, Lovisi, Santos, Legay, Abelha e Valencia (2009) realizaram uma pesquisa 
epidemiológica do suicídio entre 1980 e 2006, na qual se verificou que as taxas de suicídio no país subiram de 4,4 para 5,7 mortes por ano para 100 mil habitantes, sendo que tais números indicaram um aumento de $58 \%$ entre os homens e $1 \%$ entre as mulheres. A partir dos dados, o perfil traçado pelos autores foi estar na faixa etária entre 20 e 29 anos (34,2\%), ser solteiro (44,8\%) e do sexo masculino (77,3\%).

No que tange à tentativa de diminuir o número de suicídios, Sun et al. (2005) destacaram que se deve primeiramente entender o que está por trás deste ato. Uma das justificativas para os homens cometerem o suicídio com maior frequência seria a diferença quanto às estratégias de enfrentamento escolhidas por ambos. Enquanto as mulheres tendem a apresentar estratégias mais internas e introspectivas, os homens tendem a escolher meios externos, o que os faz escolher métodos de suicídio mais violentos e, portanto, mais eficazes (Li, DiGiuseppe, \& Froh, 2006; Lovisi et al., 2009). Outras justificativas se pautam em fatores estressores laborais e no desemprego, que podem estar envolvidos com a queda do nível socioeconômico, dificuldades e sentimento de inutilidade, características de personalidade como impulsividade, dentre outras (OMS, 2000; Rabasquinho \& Pereira, 2007). Segundo Rabasquinho e Pereira (2007), as relações entre gênero e incidência de suicídio se mostram complexas e multifatoriais; sendo assim, a determinação de apenas um fator predisponente acaba por rotular erroneamente este fenômeno, por sua complexidade e pelos fatores que envolvem o homem.

Kposowa e McElvain (2006) realizaram uma pesquisa com o intuito de verificar as diferenças entre homens e mulheres no que se refere aos métodos suicidas, e a relação entre os métodos utilizados e locais de suicídio. A amostra foi composta por 643 casos, com idades variando entre 13 e 93 anos, com média de 49 anos. Do total, 503 eram do sexo masculino $(78,2 \%)$ e 140 do sexo feminino, sendo que as causas de morte foram divididas em três variáveis, quais sejam: armas de fogo, estrangulamento/enforcamento e drogas. Dos resultados, as mulheres tenderam à prática de métodos mais internalizantes (envenenamento), ao passo que homens, as externalizantes (armas e enforcamento). Assim, tal como existem preferências por métodos, acredita-se que também existam uma multiplicidade de fatores que possam interferir no ato de suicídio, alguns dos quais são discutidos a seguir.

De forma geral, alguns dos fatores de risco elencados pela literatura para o comportamento suicida são a estrutura familiar, presença de doenças crônicas, abuso de substâncias, alcoolismo, esquizofrenia, depressão e desesperança (Baptista, 2004; Beck,
Rush, Shaw \& Emery, 1982; OMS, 2000; Parente et al., 2007; Prieto \& Tavares, 2005). Ainda, o histórico de tentativa de suicídio também deve ser verificado, visto que a probabilidade de ocorrer uma tentativa de suicídio novamente é maior (Botega, Werlang, Cais, \& Macedo, 2006).

Sun et al. (2005) comentaram sobre situações "suicidogênicas", definidas como aquelas que indicam pensamentos suicidas, que podem estar associadas com a perda familiar, do emprego, problemas de saúde, ou baixa autoestima. Outros fatores associados foram os sintomas depressivos, aumento de utilização de drogas (incluindo automedicação), sinais comportamentais tais como escrever sobre o ato, dar seus bens a alguém, escutar músicas que falem sobre morte, começar a ter pequenos acidentes consecutivamente, e avisos verbais como, por exemplo, isolamento, brincar ou fazer piadas ou brincadeiras sobre o fato. Botega et al. (2006), em artigo sobre prevenção do comportamento suicida, citam a importância de reforçar os fatores protetores, visto que quanto maiores os vínculos afetivos, envolvimento religioso e percepção positiva da vida, menor a tendência suicida.

Além das questões supracitadas, um dos fatores que vem sendo relatado por pesquisadores e que possui uma grande influência sobre a decisão do sujeito de cometer o ato suicida é a mídia. Phillips (1974) realizou uma pesquisa sobre a influência da sugestionabilidade ao suicídio, analisando a frequência de comportamentos suicidas após a publicação de matérias sobre o tema, entre 1947 a 1968, em um jornal de grande circulação nos Estados Unidos. Os resultados demonstraram um avanço significativo na ideação suicida após a publicação das notícias, havendo um decréscimo apenas dois meses após sua divulgação. Baume, Rolfe e Clinton (1998) discutiram a respeito dessa influência da mídia, e utilizaram o termo comportamental suicide modelling para nomeá-la, indicando que os atos suicidas possam ser, em grande parte, fruto do que as pessoas veem como modelos em suas vidas, principalmente na população de 15 a 24 anos, que é uma faixa etária que demonstra ser mais vulnerável ao suicídio e também às influências da mídia. Dessa forma, os autores colocaram que os programas de televisão e a imprensa, por exemplo, podem contribuir de forma a encorajar o sujeito a considerar o ato suicida como uma solução viável, baseando-se nos relatos e imagens observadas.

Thompson (1999), em artigo de revisão, discutiu acerca dos efeitos da internet no suicídio, expressando uma preocupação com relação aos jovens, por sua maior vulnerabilidade e por serem os que mais 
utilizam a internet como meio de busca de dados e de comunicação. Similarmente, Becker e Schimidt (2005) comentaram sobre a alta prevalência de suicídio entre adolescentes e o aumento de condutas suicidas de pessoas relacionadas ao impacto da mídia. A internet, tal como os autores apontaram, é uma ferramenta de grande utilização, sendo um dos principais meios que os adolescentes utilizam para se comunicar.

Percebe-se, assim, que a mídia, independente da forma de apresentação, seja por livros, jornais, revistas ou a internet, pode influenciar na decisão de cometer ou não o suicídio (Baume, Cantor, \& Rolfe, 1997; Phillips, 1974; Thompson, 1999). Conforme dados obtidos pela Internet World Stats (2009), um site dedicado a estatísticas envolvendo a internet, a América do Sul corresponde a 7,9\% de todos os usuários, sendo que o Brasil se destaca em primeiro lugar, com quase $50 \%$ do número total de usuários. Já segundo uma pesquisa realizada na ferramenta Google Trends sobre a frequência de procura da palavra "suicide" nesta ferramenta de busca, verificou-se que, desde 2004, o termo recebeu "picos de busca", ocorrendo o mesmo fato no início do ano de 2005 e no final de 2007, e tendo um novo salto em 2008, coincidente com a crise econômica mundial.

Além disso, percebe-se que atualmente é grande o número de sites voltados para o suicídio. Baume et al. (1997) pesquisaram a palavra "suicídio" na internet e observaram 130 mil resultados que variavam em categorias, a saber: sites de prevenção, culturais (identidades conhecidas que completaram o suicídio, jogos e músicas); notícias /group sites (sites de atualizações, fóruns); e mailing lists (similar ao anterior, diferindo apenas pelo fato de requisitar licença para sua utilização). Mehlum (2000), digitando a palavra "suicide" na página de buscas Altavista, em janeiro de 2001, obteve como resultado mais de um milhão de páginas, resultado considerado pelo autor como suficiente para argumentar que o termo tem sido citado em uma vasta gama de documentos.

Quando colocado em um site de busca em alemão, o mesmo termo resultou em 30 mil páginas. Quando pesquisada em português, observou-se um movimento muito semelhante, sendo que as cidades que mais retornaram resultados foram Brasília, Campinas, Florianópolis, Fortaleza e Belo Horizonte. Interessante destacar que a palavra em português foi pesquisada tanto no Brasil quanto em Portugal, no Reino Unido, Espanha, Alemanha, Japão e Estados Unidos. Entretanto, não se pode deixar de considerar a possibilidade de, por exemplo, alguns estrangeiros viverem no país ou da recíproca ser possível (Mehlum, 2000).
Becker, Meyer, Nagenborg, El-Faddagh e Shimidt (2004) debateram sobre essa problemática em um estudo de caso de uma adolescente de 17 anos que, frente ao término de relacionamento amoroso, buscou no suicídio uma saída. Após consultar sites da internet, conseguiu motivos para reforçar o comportamento e, ainda, um estímulo, uma vez que um site trazia a possibilidade do envio de remédios necessários para que ela concretizasse o ato. Os autores comentaram que a tentativa foi realizada por duas vezes, e que restaram na adolescente sentimentos como vergonha dos pais e amigos e a sensação de alívio por estar viva. Outro artigo a respeito da relação entre suicídio e mídia também expôs casos em que a internet mostrouse como ferramenta principal para tais tipos de atos, principalmente entre adolescentes (Baume et al., 1997).

Alao, Sodelberg, Pohl e Alao (2006), em artigo de revisão, discutiram a dimensão da internet na ideação suicida e a disseminação da prática da medicina em sites, o que acaba por proporcionar, na visão dos autores, que tais informações sejam utilizadas de forma negativa e assim, pró-suicídio. Os autores descreveram nove artigos cujas formas de ideação suicida se basearam em conselhos fornecidos por sites da web, muitos dos quais forneciam formas de ingerir medicamentos, certificados de morte, fotos e notas, além de facilitar a possibilidade de um grupo combinar e agendar o suicídio e, assim, ocorrer de forma coletiva. Tal como nos estudos supracitados, os autores observaram a grande quantidade de adolescentes que cometeram o ato suicida e, ainda, entre eles, o alto número dos que buscavam sites como forma de apoio antes de concretizarem o ato.

Por fim, destaca-se o estudo de Biddle, Donovan, Hawton, Kapur e Gunnell (2008) visando verificar o impacto da internet no comportamento suicida. Para tal, foram utilizadas quatro ferramentas de pesquisa, sendo elas Google ${ }^{\circledR}, \quad \operatorname{Yahoo}{ }^{\circledR}, M S N \AA$ e $A s k \AA$, a partir das quais analisaram os 10 primeiros itens dos resultados de cada base de dados, resultando em 480 páginas. Destas, foram retiradas as repetidas, restando 240 sites. Os autores realizaram as buscas em maio de 2007, utilizando 12 expressões, quais sejam: suicide; suicide methods; suicide sure methods; most effective methods of suicide; methods of suicide; ways to commit suicide; how to commit suicide; how to kill yourself; easy suicide methods; best suicide methods; pain-free suicide, e quick suicide.

Cada resultado da busca foi direcionado para um dos 14 grupos de critérios utilizados, tal como apresentado no Quadro 1. Os três primeiros grupos se relacionaram a sites dedicados ao suicídio, de 
conteúdo pró-suicídio, descrevendo métodos (sem encorajar) e descrevendo em termos elegantes. Outros três grupos envolviam conteúdo informativo sobre métodos e considerando o tema como "divertido", não sendo levado a sério. Os outros critérios usados na pesquisa envolviam sites contra o suicídio; sites de prevenção ou suporte; sites acadêmicos ou de polícia; notícias; conteúdos de bate-papo; e também páginas não encontradas ou de conteúdo não relevantes; dentre outros (Biddle et al., 2008).

\section{Procedimentos}

Baseando-se na pesquisa de Biddle et al. (2008), foram utilizadas 12 expressões-chave do estudo original, traduzidas livremente para o português e indicadas sem o uso de aspas, quais sejam (a) suicídio, (b) métodos de suicídio, (c) métodos eficientes de suicídio, (d) métodos de suicídio mais efetivos, (e) métodos suicidas, (f) modos de cometer suicídio, (g) como cometer suicídio, (h) como se matar, (i) métodos

\section{Quadro 1. Critérios utilizados por Biddle et al. (2008)}

\begin{tabular}{|l|l|}
\hline $\mathbf{N}^{\mathbf{o}}$ & \multicolumn{1}{|c|}{ Critérios de divisão dos artigos analisados por Biddle et al. (2008) } \\
\hline 1 & Conteúdos pró-suicídio, encorajando, promovendo ou facilitando-o \\
\hline 2 & Descrevendo métodos, mas sem encorajar ao ato \\
\hline 3 & Retratando suicídio em termos da moda ou estiloso (fashionable terms) \\
\hline 4 & Forneciam informações factuais sobre métodos \\
\hline 5 & $\begin{array}{l}\text { Sites que em parte brincavam sobre o assunto ou usavam expressões com linguagem pejorativa, mas com } \\
\text { sugestões de métodos reais }\end{array}$ \\
\hline 6 & Que consideram o tema divertido, não levado a sério, sendo a maioria dos métodos apresentados não reais \\
\hline 7 & Sites contra o suicídio \\
\hline 8 & Sites de prevenção ou suporte \\
\hline 9 & Sites acadêmicos ou de polícia \\
\hline 10 & Páginas não encontradas ou com termos não relevantes para a pesquisa \\
\hline 11 & Notícias de suicídios individuais ou em grupo \\
\hline 12 & Salas de bate-papo instantâneo ou fóruns de bate-papo ou debate focados em métodos suicidas' \\
\hline 13 & Salas de bate-papo instantâneo ou fóruns de bate-papo discutindo questões gerais relativas ao suicídio \\
\hline 14 & Sites com temas da atualidade, relacionados ao suicídio \\
\hline
\end{tabular}

Os resultados encontrados na pesquisa de Biddle et al. (2008) elucidaram o grande número de sites que fornecem informações pró-suicídio como, por exemplo, técnicas e/ou métodos que auxiliam aquele com ideação suicida a realizar o ato. Entretanto, não foram discutidas pelos autores as possibilidades benéficas que a internet pode trazer à pessoa com ideação, como oferecer informações técnicas, prevenir ou oferecer apoio, o que torna as pesquisas voltadas para esta temática de suma importância para a tomada de consciência de órgãos reguladores. Apesar do grande número de estudos voltados para as relações entre mídia e suicídio, percebeu-se no Brasil a falta de estudos na área. Dessa forma, o presente estudo teve como objetivo analisar, tomando o artigo supracitado como base, os conteúdos relacionados à temática do suicídio nos sites brasileiros mais acessados. fáceis de suicídio, (j) melhores métodos de suicídio, (k) suicídio indolor e (l) suicídio rápido.

A terceira expressão-chave utilizada pelos autores - "suicide sure methods" - gerou confusão em sua tradução, pela gama de sinônimos que a palavra "sure" pode indicar. Dessa forma, para esta pesquisa foi selecionada a expressão "métodos eficientes de suicídio" ao invés de "métodos certos de suicídio", conforme seria a tradução literal. Ainda com base na pesquisa de Biddle et al. (2008), foram utilizadas também as mesmas 14 categorias, tendo sido acrescidas cinco, quais sejam (15) "sites de conteúdo religioso", (16) "sites mantidos por psicólogos ou profissionais de saúde", (17) "sites sobre suicídio assistido", (18) "sites com depoimentos de quem tentou" e (19) "notícias relacionadas a suicídio, sem relato de casos". 
Foram excluídos das análises os sites repetidos ou relacionados entre si, ou seja, páginas com conteúdos de informações divididas em mais de um endereço. De acordo com o conteúdo, verificou-se se os textos eram independentes ou pertencentes a um mesmo autor. Dessa forma, diferentes textos em um mesmo site ou provedor foram considerados como diferentes, desde que os conteúdos fossem diferentes. Pela mesma razão, sites de diferentes provedores ou endereços contendo o mesmo conteúdo foram considerados repetidos.

Investigaram-se as três ferramentas de busca mais comuns da internet no Brasil, que foram Google ${ }^{\circledR} \quad$ (www.google.com.br), Yahoo!Cadê ${ }^{\circledR}$ (www.cade.com.br) e Bing ${ }^{\circledR}$, ferramenta de pesquisa

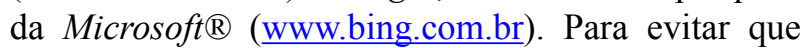
sites internacionais fossem selecionados, optou-se pelos recursos "Apenas do Brasil", na ferramenta Bing ${ }^{\circledR ;}$; "páginas do Brasill”, no Google ${ }^{\circledR}$; e "apenas páginas em português" na ferramenta Yahoo!Cadê ${ }^{\mathbb{}}$. O levantamento das páginas foi realizado no dia 22 de setembro de 2009.

\section{Resultados}

Considerando-se as 10 primeiras indicações de cada pesquisa, foram retornados, ao todo, 360 resultados. Retirando-se os repetidos e os relacionados, ou seja, as páginas com conteúdo de continuação de outras anteriormente encontradas, foram analisados os conteúdos de 209 sites. Deste total, 28,8\% ( $\mathrm{n}=63)$ se enquadraram no critério 10 , ou seja, página não encontrada, fora do ar, ou com conteúdo não relevante para a pesquisa, não relacionado ao tema. Foram adicionadas a esta categoria, além das sem relação com o tema, aquelas páginas com notícias ou comentários sobre homens-bomba, kamikazes japoneses e depressão, por exemplo, por não se envolverem diretamente com propósito do artigo. Assim, somente foram considerados os 146 resultados relevantes encontrados.

A partir da análise dos conteúdos, 10 sites foram incluídos em mais de uma categoria. Isso ocorreu com as páginas em que o conteúdo possuía uma divisão "meio-a-meio" de cada categoria. Por exemplo, sites de provedores religiosos, com direcionamento religioso à temática do suicídio, mas que continham também informações de outros sites, discussão geral sobre o tema, independente do fator religiosidade, poderiam ser categorizados tanto como "conteúdo religioso" quanto "temas atuais relacionados". Dessa forma, as análises referentes às categorias utilizadas no estudo levam em consideração 156 sites.
Inicialmente, compararam-se os critérios encontrados no artigo original e para o levantamento de sites no Brasil. Não foram encontrados sites com conteúdo pró-suicídio - promovendo, encorajando ou facilitando-o, ao contrário da pesquisa de Biddle et al. (2008), na qual $50 \%$ dos 90 sites dedicados ao suicídio continham tal conteúdo. Apesar disso, deve-se atentar que $3,2 \%(n=5)$ traziam descrição de métodos, mesmo sem encorajamento e que $2,6 \%(n=4)$ eram chats ou fóruns focados na discussão de métodos, sendo que um destes fóruns possuía informações detalhadas para vários tipos de métodos, com prós e contras de cada um, além de comentários sobre métodos de morte mais rápida ou indolor. Não foram categorizados sites com conteúdo factual sobre métodos, tendo sido encontradas descrições detalhadas sobre métodos somente nas páginas com o formato de chats ou fóruns de discussão. Isso talvez demonstre que os brasileiros possuem preferências diferentes dos ingleses sobre o tipo de sites para buscarem informações, dando preferências a chats e/ou fóruns de discussão.

Do total de endereços avaliados, 7,53\% referiam-se ao artigo original de Biddle et al. (2009), em forma de notícia ou de comentários sobre os resultados daquela pesquisa, geralmente sob o título "sites que encorajam suicídio são os mais fáceis de encontrar na web", sendo que as bases de dados Google ${ }^{\circledR}$ e Yahoo!Cadê ${ }^{\circledR}$ retornaram o maior número de resultados. Além disso, três sites faziam referência direta ao livro "Manual completo de suicídio", do escritor japonês Wataru Tsurumi, que discute diferentes tipos de métodos, avaliando-os quanto à letalidade, dor, preparação necessária para o ato $\mathrm{e}$ aparência do corpo pós-morte.

Pôde-se perceber que a maioria dos resultados categorizados $(21,8 \%)$ fazia referência a sites acadêmicos, isto é, àqueles com pesquisas empíricas ou bibliográficas, publicadas em revistas de acesso livre ou não, locais ou nacionais, artigos, publicações de trabalhos de conclusão de curso ou similares, incluindo resumos (Tabela 1). Em seguida, estão os sites com temas da atualidade, ou seja, blogs pessoais ou sites contendo textos, comentários, resumos de livros sobre o assunto e informações atuais relacionadas ao tema. Algumas dessas páginas traziam, inclusive, dados epidemiológicos, fatores de risco e possíveis causas, mas não foram categorizados como acadêmicos por não se tratarem de estudos, mas de informações gerais. Como já explicitado, não foram encontrados artigos de conteúdo prósuicídio encorajando, promovendo ou facilitando o ato (categoria 1) e sites que forneciam informações factuais sobre métodos (categoria 2). 
Tabela 1. Distribuição da frequência em relação às categorias

\begin{tabular}{|c|l|c|c|}
\hline Categoria & \multicolumn{1}{|c|}{ Descrição da categoria } & f & \% \\
\hline 9 & Acadêmicos & 34 & 21,8 \\
\hline 14 & Sites com temas da atualidade, relacionados ao suicídio & 19 & 12,2 \\
\hline 15 & Sites de conteúdo religioso & 15 & 9,6 \\
\hline 3 & Retrata suicídio em termos elegantes & 14 & 9,0 \\
\hline 16 & Sites mantidos por psicólogos ou profissionais de saúde & 11 & 7,1 \\
\hline 8 & Prevenção ou suporte & 9 & 5,8 \\
\hline 11 & Notícias de suicídios individuais ou em grupo & 8 & 5,1 \\
\hline 13 & $\begin{array}{l}\text { Salas de bate-papo instantâneo ou fóruns de bate-papo discutindo questões } \\
\text { gerais relativas }\end{array}$ & 8 & 5,1 \\
\hline 19 & Notícias relacionadas a suicídio, sem relato de casos & 8 & 5,1 \\
\hline 5 & $\begin{array}{l}\text { Sites que brincam, em parte, com linguagem pejorativa, mas com sugestões de } \\
\text { métodos reais }\end{array}$ & 6 & 3,8 \\
\hline 2 & Descrição de métodos, sem encorajar & 5 & 3,2 \\
\hline 7 & Sites contra suicídio & 5 & 3,2 \\
\hline 12 & $\begin{array}{l}\text { Salas de bate-papo instantâneo ou fóruns de bate-papo ou debate focados em } \\
\text { métodos suicidas }\end{array}$ & 4 & 2,6 \\
\hline 18 & Sites com depoimentos de quem tentou & 4 & 2,6 \\
\hline 6 & Consideram o tema divertido, não levado a sério, com métodos falsos & 3 & 1,9 \\
\hline 17 & Sites sobre suicídio assistido & 3 & 1,9 \\
\hline & Total & $\mathbf{1 5 6}$ & $\mathbf{1 0 0}$ \\
\hline
\end{tabular}

Outro tipo de site muito frequente nos resultados brasileiros foram os de conteúdo religioso $(9,6 \%)$, assunto que não foi discutido no artigo original. Estes sites geralmente continham discursos de apoio às pessoas com ideação suicida, chamando atenção para aspectos bíblicos e justificando que "somente Deus pode decidir sobre a vida de uma pessoa" e que por isso, a morte não seria a solução. Em outros sites foram observadas informações globais como epidemiologia e fatores de risco, mas com direcionamento a questões religiosas.

Pôde-se perceber também uma frequência razoável de sites mantidos por psicólogos ou profissionais de saúde, com conteúdo diversificado, informando fatores de risco e dados epidemiológicos retirados da página da OMS, e também de diferentes livros, totalizando aproximadamente $7 \%$ dos sites analisados. Observou-se ainda que 9\% $(n=14)$ dos conteúdos retrataram o suicídio em termos elegantes, ou seja, aqueles contendo poesias, letras de música, comentários sobre cenas de filmes e que encaram o suicídio como algo estiloso (fashionable terms).

No outro extremo - o que pode ser considerado um aspecto positivo - somente três sites possuíam conteúdo que consideram o tema como algo divertido, não levado a sério, chegando a apresentar métodos falsos, em tom de piada. Por exemplo, em um destes sites havia comentários sobre diferentes métodos de suicídio, sendo o casamento o primeiro da lista. Também foi baixo o número de sites contendo informações, relatos de caso ou histórias de suicídio assistido (1,9\%), geralmente comentando sobre os casos clássicos. Interessante destacar que as salas de bate-papo instantâneo ou fóruns discutindo questões gerais de suicídio e aqueles focados em discussão sobre métodos apresentaram diferenças. Enquanto 5\% discutiam questões gerais, comentários sobre notícias ou sobre os conteúdos divulgados na internet sobre suicídio, aproximadamente a metade estava focalizada em discutir métodos. 
No que concerne às ferramentas de pesquisas utilizadas, a Google foi a que retornou o maior número de resultados, totalizando $37 \%(\mathrm{n}=54)$. Em seguida, Yahoo! Cadê ${ }^{\circledR}, \operatorname{com} 34,2 \%(\mathrm{n}=50)$ e Bing ${ }^{\circledR}$, com $28,8 \%(n=42)$ do total de artigos analisados. Pôde ser verificado como cada expressão-chave se comportou para cada base de pesquisas utilizada. As expressões "suicídio" e "métodos de suicídio" tiveram maior retorno pela ferramenta Bing ${ }^{\circledR}$, enquanto o Google ${ }^{\circledR}$ apresentou um maior número de sites para os termos de busca que se relacionam especificamente a métodos (Tabela 2).

\section{Discussão e conclusão}

O suicídio é considerado pela Organização Mundial de Saúde como um sério problema de saúde pública, sendo a terceira causa de morte mais frequente na população adulta e jovem (OMS, 2000; WHO, 2003). Alguns autores chamam a atenção para a alta prevalência de suicídio entre adolescentes e jovens, ressaltam o impacto da mídia nessas populações e discutem o efeito da internet sobre o fenômeno suicida (Becker \& Schimidt, 2005; Thompson, 1999). Segundo tais autores, por um lado, a internet é uma ferramenta

Tabela 2. Frequência de resultados apresentados para cada termo, por ferramenta de busca e no total

\begin{tabular}{|l|c|c|c|c|c|}
\hline & \multicolumn{3}{|c|}{ Ferramenta de busca } & \multirow{2}{*}{ Total } & \multirow{2}{*}{$\%$} \\
\cline { 2 - 4 } & Bing & Google & Yahoo & & \multirow{2}{*}{ Métodos eficientes de suicídio } \\
\hline Suicídio & 5 & 7 & 5 & 17 & 11,6 \\
\hline Métodos de suicídio & 7 & 4 & 4 & 15 & 10,3 \\
\hline Suicídio indolor & 4 & 5 & 6 & 15 & 10,3 \\
\hline Modos de cometer suicídio & 3 & 5 & 6 & 14 & 9,6 \\
\hline Suicídio rápido & 5 & 4 & 4 & 13 & 8,9 \\
\hline Como cometer suicídio & 1 & 8 & 3 & 12 & 8,2 \\
\hline Métodos de suicídio mais efetivos & 1 & 6 & 4 & 11 & 7,5 \\
\hline Métodos suicidas & 2 & 6 & 3 & 11 & 7,5 \\
\hline Métodos fáceis de suicídio & 2 & 2 & 5 & 9 & 6,2 \\
\hline Melhores métodos de suicídio & 3 & 3 & 3 & 9 & 6,2 \\
\hline Como se matar & 0 & 2 & 2 & 4 & 2,7 \\
\hline Total & $\mathbf{4 2}$ & $\mathbf{5 4}$ & $\mathbf{5 0}$ & $\mathbf{1 4 6}$ & $\mathbf{1 0 0 , 0}$ \\
\hline
\end{tabular}

Por outro lado, as expressões "melhores métodos" e "suicídio rápido" tiveram um retorno de resultados de maneira semelhante para as três ferramentas de pesquisas utilizadas. De maneira geral, as expressões-chave que mais retornaram páginas relacionadas ao tema foram "métodos eficientes de suicídio", "suicídio", "métodos de suicídio" e "suicídio indolor", sendo que "como cometer suicídio" retornou o menor número de resultados, apenas $2,7 \%$. de alta utilização, sendo um dos meios mais utilizados para busca de informações e comunicação informal e, por outro lado, os adolescentes se tornam mais vulneráveis às diversas informações que circulam na rede.

Pensando nisto, o presente artigo teve como objetivo a adaptação do estudo de Biddle et al. (2008) visando analisar quais os conteúdos relacionados ao suicídio mais acessados por brasileiros. Observou-se que, diferente do artigo replicado e do encontrado 
em outros países em pesquisas supramencionadas (Becker \& Schidt, 2005; Phillips, 1974; Thompson, 1999), no Brasil inexistem sites que encorajam ou facilitam o ato suicida. Tal resultado, entretanto, não deve ser interpretado de forma a significar que os brasileiros não acessam ou não procuram saber sobre o suicídio. O Brasil é o primeiro no ranking da América Latina a pesquisar o termo "suicídio", o que pode indicar que por mais que não existam sites prósuicídio, tais informações são encontradas em outras páginas internacionais sobre o tema (Google Trends, 2009).

Outro ponto a ser considerado é a influência que estas páginas brasileiras possuem sobre $o$ comportamento suicida. A existência de sites que sejam contra o suicídio pode ser um fator que auxilie na queda de números de suicídios; entretanto, este dado é difícil de ser analisado, considerando que ele possa ser bidirecional, ou seja, a inexistência de sites que incentivem pode indicar tanto um fator preventivo (a leitura das páginas podem diminuir a ideação suicida) quanto uma variável que pode potencializar a procura e a criação e fóruns e chats sobre o tema.

Além disso, deve-se levar em consideração que o número de fóruns acessados no Brasil é aparentemente maior do que em outros países, conforme pesquisa realizada no Google. Tal indicativo pode denotar que o comportamento do brasileiro é mais instantâneo, frente a suas preferências, e se pauta, de um modo geral, mais nas discussões em fóruns ou batepapos, do que no desenvolvimento ou acesso de páginas estáticas para obter informações. Ainda, esta informação faz com que outras possibilidades, que fogem ao escopo do presente estudo, possam ser cogitadas, levantando à necessidade da realização de um estudo de perfil do usuário brasileiro para melhores conclusões a respeito.

Dessa forma, por mais que não tenham sido encontrados muitos sites voltados para o encorajamento do suicídio, tais fóruns de discussão podem servir para tal finalidade. A presença de fóruns que demonstram maior rapidez das informações também pode implicar na menor acessibilidade ao fórum após a divulgação de um ato suicida, considerando que geralmente estas páginas são excluídas da rede e impedidas de continuarem a ser exibidas. . Assim sendo, o número estipulado de páginas não encontradas pode estar diretamente associado com esses fóruns e também à lacuna existente entre os suicídios cometidos principalmente entre jovens e a influência deste meio de comunicação.

Outro aspecto discrepante em relação ao artigo original de Biddle et al. (2008) refere-se à presença de sites com conteúdos caracterizados como religiosos, com ou sem relatos de casos. Botega et al. (2006) apontaram o envolvimento religioso como um fator protetivo à ideação suicida. Desta forma, hipotetizase que os dados dos sites religiosos possam fornecer informações que desencorajem aqueles que procuram uma solução para seus problemas no ato suicida uma vez que os sites encontrados com tal conteúdo se apresentam com a intenção de apoiar as pessoas desesperançosas, com desejo de morte; portanto, não encorajam o ato, mas ao contrário, demonstram opinião contrária.

Além disso, foram também encontrados sites mantidos por profissionais da área de saúde, como por exemplo, psicólogos. Alao et al. (2006), discutindo o papel da internet sobre o comportamento suicida, afirmaram que embora a internet tenha seus fatores positivos como meio de comunicação, pode gerar disseminação indiscriminada e distorcida de informações, o que contribui para o uso indevido de seu conteúdo. Entretanto, quando esse conteúdo apresenta embasamento de um profissional, o que é divulgado tem menor chance de ser distorcido.

Embora tenha sido apontado pelo estudo que o Google foi a ferramenta de busca com maior número de resultados e que os sites brasileiros tiveram baixa frequência de páginas encorajando o suicídio, é preciso considerar tais resultados com cautela, pois estão envolvidos diferentes fatores. Alguns deles envolvem a forma como cada ferramenta de busca inclui e exclui sites em seus resultados de pesquisa, e como funcionam as restrições de conteúdo divulgadas por cada ferramenta. Além disso, vários autores chamam a atenção para o número crescente de sites voltados para o suicídio (Baume et al., 1997; Mehlum, 2000). Assim, atenta-se para a necessidade de realização de pesquisas que estudem a influência da divulgação da mídia em seus diversos aspectos, e como tal divulgação pode afetar o comportamento, da população em geral e não somente daquelas predispostas ao suicídio.

Uma vez que esta pesquisa apresenta algumas limitações, sugere-se que em próximos estudos haja um maior controle do número de casos de suicídio por cidades e regiões que tiveram associações com a influência da mídia, a discriminação entre os sexos, de forma a verificar qual o gênero que busca maiores informações na internet sobre este tema. E, por fim, um maior controle a respeito das faixas etárias, com a finalidade averiguar o que empiricamente vem sendo mostrado, ou seja, que a faixa etária de 15 a 24 anos é aquela na qual os casos de suicide modelling são mais detectados (Baume et al., 1998; Becker \& Schimidt, 2005; Thompson, 1999). 
Finalmente, comparando aos resultados da pesquisa de Biddle et al. (2008), verifica-se que, por mais que os procedimentos adotados tenham sido os mesmos, os resultados alcançados foram diferentes, indicando outro perfil de comportamento do brasileiro em comparação ao estrangeiro frente a tais temas. Esse fato aponta para a importância de serem consideradas as diferenças culturais ao se estudar a influência da mídia no ato suicida, principalmente porque o perfil das informações traz muito do que a sociedade aceita, suas crenças e costumes. Outro ponto é a necessidade de criação de políticas públicas para essa finalidade, bem como o desenvolvimento de páginas oficiais que abordem o tema do suicídio e se voltem à busca do que está por trás do fenômeno suicida, tal como apresentado por Sun et al. (2005). Os trabalhos voltados à promoção de saúde produzem impactos positivos, principalmente sobre aqueles que já tentaram se matar em algum momento da vida, uma vez que podem ser grandes as chances de recaída.

\section{Referências}

Alao, A. O., Soldberg, M., Pohl, E. L., \& Alao, A. L. (2006). Cybersuicide: Review the role of internet on suicide. Cyber psychology and behavior, 9(4), 489-493.

Andriessen, K. (2006). Do we need to be cautions in evaluating suicide statistics? European Journal of Public Health, 16(4), 445-447.

Baptista, M. N. (2004). Suicídio: Aspectos Teóricos e Pesquisas Internacionais. In M. N. Baptista (Org.), Suicídio e depressão: atualizações (pp. 3-23). Rio de Janeiro: Guanabara Koogan.

Baume, P., Cantor, C. H., \& Rolfe, A. (1997). Cybersuicide: The role of interaptive suicide notes on the internet. Crisis, 18(2), 73-79.

Baume, P., Rolfe, A., \& Clinton, M. (1998). Suicide on the internet: A focus for nursing intervention? Australian and New Zeland Journal of Mental Health Nursing, 7, 134-141.

Beck, T. A., Rush, J. A., Shaw, F. B., \& Emery, G. (1982). Terapia cognitiva da Depressão. Rio de Janeiro: Zahar.

Becker, K. \& Schmidt, M. H. (2005). When kids seek help on line: Internet chat rooms and suicide. Reclaming child and youth, 13(4), 229-230.

Becker, K., Meyer, M., Nagenborg, M., El-Faddagh, M., \& Shmidt, M. H. (2004). Parasuicide on line: Can suicide websites trigger suicidal behaviours in predisposed adolescents? Nord Journal of Psychiatry, 58, 111- 114.

Biddle, L., Donovan, J., Hawton, K., Kapur, N., \& Gunnel, N. (2008). Suicide and the internet. BMJ, 336(12), 800-802.

Botega, N. J., Werlang, B. S. G., Cais, C. F. S., \& Macedo, M. M. K. (2006). Prevenção do comportamento suicida. Psico, 37(3), 213-220.

Corcoran, P., Arensman, E., \& O'Mahony, D. (2006). Suicide other external-cause mortality statistics in Ireland: a comparison of registration and occurrence data. Crisis, 27(3), 130-134.

Google Trends. (2009). Ferramenta de análise de termos de pesquisa do Google. Acesso em 13 de novembro, 2009, em http://www.google.com/trends
Internet World States. (2010). Estatísticas de usuários da internet. Acesso em 20 de dezembro, 2009, em http://www. internetworldstats.com

Kliemann, D. V. O. (2007). Estudo epidemiológico de óbitos por suicídio na região da grande Florianópolis de 1991 a 2005. Trabalho de Conclusão de Curso, Universidade Federal de Santa Catarina, Florianópolis. Acesso em 10 de dezembro, 2009, em http://www.bibliomed.ccs.ufsc.br/CM0614.pdf

Kposowa, A. J. \& McElvain, J. P. (2006). Gender, place and method of suicide. Society of Psychiatry Epidemiology, 41, 435-443.

Li, C. E., DiGiuseppe, R., \& Froh, J. (2006). The roles of sex, gender and coping in adolescent depression. Adolescence, 41(163), 409-415.

Lovisi, G. M., Santos, S. A., Legay, L., Abelha, L., \& Valencia, L. (2009). Análise epidemiológica do suicídio no Brasil entre 1980 e 2006. Revista Brasileira de Psiquiatria, 31(2), 586-594.

Mehlum, L. (2000). The internet, suicide and suicide prevention. Crisis, 21(4), 186- 188.

Mello-Santos, C., Bertolote, J. M., \& Wang, Y-P. (2005). Epidemiology of suicide in Brazil (1980-2000): Characterization of age and gender rates of suicide. Revista Brasileira de Psiquiatria, 27(2), 131-134.

Organização Mundial da Saúde - OMS. (1993). Classificação Internacional de Doenças - CID10. Porto Alegre: Artes Médicas.

Organização Mundial da Saúde - OMS. (2000). Prevenção do suicídio: um manual para médicos clínicos gerais. Acesso em 20 de outubro, 2009, em www.whqlibdoc.who.int/ publications/2000/WHO_MNH_MBD 00 1 po.pdf

Parente, A. C. M., Soares, R. B., Araújo, A. R. F., Cavalcante, I. S., Monteiro, C. F. S. (2007). Caracterização dos casos de suicídio em uma capital do nordeste brasileiro. Revista Brasileira de Enfermagem, 60(4), 377-381.

Phillips, D. P. (1974). The influence of suggestion on suicide: Substantive and theoretical implications of the werther effect. American Sociological Review, 39, 340-354.

Prieto, D. \& Tavares, M. (2005). Fatores de risco para suicídio e tentativa de suicídio: incidência, eventos estressores $e$ transtornos mentais. Jornal Brasileiro de Psiquiatria, 54(2), 146-154.

Rabasquinho, C. \& Pereira, H. (2007). Gênero e saúde mental: uma abordagem epidemiológica. Análise Psicológica, 3(15), 439-454.

Sadock, B. J. \& Sadock, V. A. (2007). Transtornos da alimentação na primeira infância. In B. J. Sadock \& V. A. Sadock, Compêndio de Psiquiatria: Ciência do comportamento e Psiquiatria Clínica (pp. 1228-1234). São Paulo: Artmed.

Sun, F. K., Long, A., Boore, J., \& Tsao, I. (2005). Suicide: A literature review and its implications for nursing practice in Taiwan. Journal of Psychiatric and Mental Health Nursing, 12, 447- 455 .

Thompson, S. (1999). The Internet and its potential influence on suicide. Psychiatric Bulletin, 25, 449-451.

World Health Organization - WHO. (2000a). Preventing suicide: A resource for general physicians. Mental and Behavioral Disorders. Acesso em 20 de outubro, 2009, em http://www.who.int/mental_health/media/en/56.pdf

World Health Organization - WHO. (2000b). Prevention for suicidal behaviors: A task for all. Mental and Behavioral 
Disorders. Acesso em 20 de outubro, 2009, em http://www. healthinternetwork.com/mental_health/prevention/suicide/ information/en

World Health Organization - WHO. (2003). Suicide prevention. Mental Health. Acesso em 08 de outubro, 2009, em http://www.who.int/mental health/prevention/suicide/ suicideprevent/en

Recebido em: 14/09/2010

Revisão em: 20/04/2012

Aceite em: 14/06/2012

Juliana Oliveira Gomes é Doutora em Psicologia pela Universidade São Francisco, na área de Avaliação Psicológica em Contextos da Saúde Mental. Docente do curso de Psicologia e membro do Laboratório de Análises e Medidas em Psicologia (LAMP) da Faculdade Estácio de Sá, Núcleo Zona da Mata, Juiz de Fora/MG. Docente do curso de Psicologia da Faculdade de Minas, FAMINASMuriaé/MG. Endereço: Faculdade Estácio de Sá, Curso de Psicologia. Estrada Presidente João Goulart, 600. Cruzeiro Sul. Juiz de Fora/MG, Brasil. CEP 36030-120. E-mail: julianaoliveiragomes@yahoo.com.br

Makilim Nunes Baptista é Doutor pelo Departamento de Psiquiatria e Psicologia Médica da Escola Paulista de Medicina. Docente do Programa de Pós- Graduação Stricto Sensu em Psicologia da Universidade

São Francisco, Itatiba, São Paulo. Coordenador do Laboratório de Avaliação Psicológica em Saúde Mental (LAPSAM-III). Membro do Grupo de Trabalho de Família da ULAPSI - União Latino-Americana de Entidades de Psicologia. Bolsista de Produtividade em Pesquisa pelo CNPq. E-mail: makilim01@gmail.com

Adriana Munhoz Carneiro é Psicóloga. Mestre em Psicologia pela Universidade São Francisco, na área de Avaliação Psicológica em Contextos da Saúde Mental.

Discente do programa de Pós-Graduação Lato Sensu do Instituto de Psiquiatria da Faculdade de Medicina da Universidade de São Paulo, cursando Especialização em Terapia Cognitivo Comportamental em Saúde Mental. Membro do Grupo de Distúrbios Afetivos do Hospital das Clínicas/FM/USP. E-mail: adrianacarneiro01@gmail.com

Hugo Ferrari Cardoso é Psicólogo, Doutor em Psicologia pela Universidade São Francisco, na área de Avaliação Psicológica em Contextos da Saúde Mental. Docente dos cursos de Psicologia da Universidade Sagrado Coração, Bauru/SP e da Fundação Educacional "Dr Raul Bauab", Jaú/SP. Coordenador do curso de Pós-graduação Lato Sensu - Especialização em Psicologia do Trânsito da Universidade Sagrado Coração. E-mail: hfcardoso@gmail.com

\section{Como citar:}

Gomes, J. O., Baptista, M. N., Carneiro, A. M., \& Cardoso, H. F. (2014). Suicídio e internet: análise de resultados em ferramentas de busca. Psicologia \& Sociedade, 26(1), 63-73. 